\title{
Selective Binding of Distamycin A Derivative to G-Quadruplex Structure $\left[\mathbf{d}(\text { TGGGGT) }]_{4}\right.$
}

\author{
Bruno Pagano, ${ }^{1}$ Iolanda Fotticchia, ${ }^{2}$ Stefano De Tito, ${ }^{3}$ Carlo A. Mattia, ${ }^{1}$ Luciano Mayol, ${ }^{3}$ \\ Ettore Novellino, ${ }^{4}$ Antonio Randazzo, ${ }^{3}$ and Concetta Giancola ${ }^{2}$ \\ ${ }^{1}$ Dipartimento di Scienze Farmaceutiche, Università di Salerno, Via Ponte don Melillo, 84084 Fisciano, Italy \\ ${ }^{2}$ Dipartimento di Chimica "P. Corradini", Università di Napoli Federico II, Via Cintia, 80126 Napoli, Italy \\ ${ }^{3}$ Dipartimento di Chimica delle Sostanze Naturali, Università di Napoli Federico II, Via D. Montesano 49, 80131 Napoli, Italy \\ ${ }^{4}$ Dipartimento di Chimica Farmaceutica e Tossicologica, Università di Napoli Federico II, Via D. Montesano 49, 80131 Napoli, Italy
}

Correspondence should be addressed to Antonio Randazzo, antonio.randazzo@unina.it and Concetta Giancola, giancola@unina.it

Received 21 January 2010; Accepted 7 March 2010

Academic Editor: Jean Louis Mergny

Copyright () 2010 Bruno Pagano et al. This is an open access article distributed under the Creative Commons Attribution License, which permits unrestricted use, distribution, and reproduction in any medium, provided the original work is properly cited.

Guanine-rich nucleic acid sequences can adopt G-quadruplex structures stabilized by layers of four Hoogsteen-paired guanine residues. Quadruplex-prone sequences are found in many regions of human genome and in the telomeres of all eukaryotic organisms. Since small molecules that target G-quadruplexes have been found to be effective telomerase inhibitors, the identification of new specific ligands for G-quadruplexes is emerging as a promising approach to develop new anticancer drugs. Distamycin A is known to bind to AT-rich sequences of duplex DNA, but it has recently been shown to interact also with G-quadruplexes. Here, isothermal titration calorimetry (ITC) and NMR techniques have been employed to characterize the interaction between a dicationic derivative of distamycin A (compound 1) and the $\left[\mathrm{d}(\mathrm{TGGGGT}]_{4}\right.$ quadruplex. Additionally, to compare the binding behaviour of netropsin and compound 1 to the same target, a calometric study of the interaction between netropsin and [d(TGGGGT) $]_{4}$ has been performed. Experiments show that netropsin and compound $\mathbf{1}$ are able to bind to $[\mathrm{d}(\mathrm{TGGGGT})]_{4}$ with good affinity and comparable thermodynamic profiles. In both cases the interactions are entropically driven processes with a small favourable enthalpic contribution. Interestingly, the structural modifications of compound $\mathbf{1}$ decrease the affinity of the ligand toward the duplex, enhancing the selectivity.

\section{Introduction}

It is well known that G-rich sequences can adopt unusual DNA secondary structures with biological significance, the G-quadruplexes. These structures are four-stranded helical complexes, composed of stacks of G-tetrads, a cyclic array of four guanine bases which are connected by Hoogsteen hydrogen bonding. The phosphodiester backbones of the four quadruplex-forming strands could be in parallel or antiparallel relative orientation, generating grooves of different width and several loops arrangement. Generally, the formation of G-quadruplexes requires the presence of metal cations that selectively bind to guanine $\mathrm{O} 6$ carbonyl groups in the central cavity generated by the stacked layers of Gtetrads $[1]$.

Sequences with propensity to form G-quadruplexes have been identified in biologically significant genomic regions such as telomeres or oncogene promoter regions [2, 3], which have emerged as potential targets for anticancer drug development. Very importantly, DNA G-quadruplex structures that form in the promoter region of oncogenes have recently showed to play a role in the control of gene expression and the modulation of such expression could be achieved by targeting these structures [4].

Telomeric sequences, which are found at the ends of eukaryotic chromosomes, consist of G-rich repeats on the single-stranded $3^{\prime}$ end. Oligonucleotides corresponding to the G-rich $3^{\prime}$ strand of telomeric DNA of a variety of organisms have been shown to fold into G-quadruplex DNA structures [5]. The truncated sequence of Tetrahymena telomeric DNA, d(TGGGGT), forms a tetramolecular quadruplex in presence of cations, with a parallel-stranded, right-handed helical structure containing four equivalent grooves [6]. 
The biological importance of telomeric G-quadruplex structures arises from the evidence that high telomerase activity (not present in somatic cells) has been implicated in about $85 \%$ of tumours [7]. The telomerase elongates the G-rich strand of telomeric DNA, leading the cancer cells to infinite lifetime. For that reason, the inhibition of telomerase has become an interesting strategy for the anticancer therapy [8]. Since the formation of G-quadruplexes by telomeric DNA inhibits the activity of telomerase, small molecules that stabilize the G-quadruplex structures could potentially be effective chemotherapeutic agents [9].

In this scenario, the identification of new ligands that are specific for G-quadruplex structures is emerging as a promising approach to develop new anticancer drugs. Despite the fact that the structures of G-quadruplexes differ considerably from the double helix, the design of selective quadruplex ligands is very difficult, because the structure of G-quadruplexes varies in several different ways, including number and orientation of strands, grooves width, and loops topology [1]. Nevertheless, a number of G-quadruplex binding agents has been proposed so far and some of these have been demonstrated to be effective telomerase inhibitors [10].

Most of the reported G-quadruplex ligands interact with the outer G-tetrads of the structures through $\pi-\pi$ stacking interactions [11]. The only groove binder experimentally proven to date has been investigated in our laboratories; it is the distamycin A that interacts in a groove-binding mode with the quadruplex $[\mathrm{d}(\mathrm{TGGGGT})]_{4}[12]$. This finding, along with the observation that derivatives of distamycin could be effective inhibitors of the human telomerase [13], has stimulated other investigations. In a previous study, we investigated the importance of the crescent shape extension by varying the pyrrole units number in distamycin A [14, 15]. We focused our attention on the interaction of two carbamoyl analogues of distamycin A, containing four and five pyrrole units, respectively. Experiments revealed that the presence of one additional pyrrole unit affects the affinity as well as the stoichiometry of the binding whereas the addition of two pyrrole units leads to a total loss of interaction between the derivative and the $[\mathrm{d}(\mathrm{TGGGGT})]_{4}$.

In this work, we evaluate the effect of a second cationic group, placed at the end of the molecule, on the interaction with DNA molecules. In particular, we report a calorimetric and NMR study of the interaction between the $[\mathrm{d}(\mathrm{TGGGGT})]_{4}$ quadruplex and a new distamycin A derivative (compound 1, Figure 1), where the initial formamide group is replaced by a charged $\mathrm{N}$-formimidoil moiety.

In addition, since compound $\mathbf{1}$ can also be considered as an analogue of netropsin because it presents one pyrrole unit more than netropsin (three instead of two), but two cationic ends like it (even if different groups), we compare the binding of compound $\mathbf{1}$ to $[\mathrm{d}(\mathrm{TGGGGT})]_{4}$ with the binding of netropsin to the same target.

Finally, to investigate the selectivity of compound $\mathbf{1}$ for the G-quadruplex relative to duplex, we also performed a study of the interaction between the drug and the selfcomplementary DNA duplex d(CGCGAATTCGCG) $)_{2}$. This symmetric dodecamer was chosen because (a) it contains the central AATT core, which is considered the specific binding site for distamycin and netropsin; (b) the interaction with netropsin is well characterized in literature, from both structural and thermodynamic point of view [16-19].

\section{Materials and Methods}

2.1. Materials. The d(TGGGGT) and d(CGCGAATTCGCG) oligonucleotide sequences used for this study were purchased from the Primm Company (Milan, Italy).

Quadruplex and duplex samples were prepared by dissolving the lyophilised compound in a buffer solution containing $20 \mathrm{mM}$ phosphate with $70 \mathrm{mM} \mathrm{KCl}, 0.1 \mathrm{mM}$ EDTA at $\mathrm{pH}$ 7.0. The resulting solutions were annealed by heating at $95^{\circ} \mathrm{C}$ for 5 minutes. The solutions were then slowly cooled to room temperature and equilibrated for 1 day at $4^{\circ} \mathrm{C}$. The concentration of oligonucleotides was determined by UV adsorption measurements at $90^{\circ} \mathrm{C}$ using molar extinction coefficient values $\varepsilon_{(260 \mathrm{~nm})}$ of 57800 and $110700 \mathrm{M}^{-1} \mathrm{~cm}^{-1}$ for $\mathrm{d}($ TGGGGT) and $\mathrm{d}$ (CGCGAATTCGCG), respectively. The molar extinction coefficients were calculated by the nearest neighbour model [20].

Compound 1 has been synthesized as reported in the literature [21], while netropsin has been purchased from Sigma-Aldrich (St. Louis, MO). Drug solutions have been prepared in the same buffer used for the oligonucleotides, and the concentration has been estimated by UV spectroscopy using the calculated extinction coefficient value $\varepsilon_{(297 \mathrm{~nm})}$ of $30000 \mathrm{M}^{-1} \mathrm{~cm}^{-1}$ for compound $\mathbf{1}$ and the reported extinction coefficient value $\varepsilon_{(296 \mathrm{~nm})}$ of $21500 \mathrm{M}^{-1} \mathrm{~cm}^{-1}$ for netropsin [22].

2.2. Nuclear Magnetic Resonance Experiments. [d(TGGGGT) $]_{4}$ and d(CGCGAATTCGCG) $)_{2}$ NMR samples were prepared at a concentration of $2 \mathrm{mM}$ and $1 \mathrm{mM}$ respectively, in $0.2 \mathrm{ml}\left(\mathrm{H}_{2} \mathrm{O} / \mathrm{D}_{2} \mathrm{O} 9: 1\right)$ buffer solution having $10 \mathrm{mM} \mathrm{KH} \mathrm{PO}_{4} / \mathrm{K}_{2} \mathrm{HPO}_{4}, 70 \mathrm{mM} \mathrm{KCl}, 0.1 \mathrm{mM}$ EDTA, and $\mathrm{pH}$ 7.0. NMR spectra were recorded with Varian Unity INOVA $700 \mathrm{MHz}$ spectrometer. ${ }^{1} \mathrm{H}$ chemical shifts were referenced relative to external sodium 2,2-dimethyl-2-silapentane-5sulfonate (DSS). 1D proton spectra of samples were recorded using pulsed-field gradient DPFGSE for $\mathrm{H}_{2} \mathrm{O}$ suppression $[23,24]$.

2.3. Isothermal Titration Calorimetry Experiments. ITC experiments were carried out at $298 \mathrm{~K}$ using a highsensitivity CSC-5300 Nano-ITC microcalorimeter from Calorimetry Science Corporation (Lindon, Utah) with a cell volume of $1 \mathrm{ml}$. Before each ITC experiment, the $\mathrm{pH}$ of each solution was checked, the reference cell was filled with deionised water, and the DNA solutions were degassed for 5 minutes to eliminate air bubbles. Care was taken to start the first addition after baseline stability had been achieved. In each titration, volumes of $5-10 \mu \mathrm{L}$ of a solution containing compound 1 or netropsin at a concentration of $600-700 \mu \mathrm{M}$ were injected into a solution of quadruplex or duplex DNA $(30 \mu \mathrm{M})$ in the same buffer, using a computer-controlled $250 \mu \mathrm{L}$ microsyringe. In order to allow the system to reach 


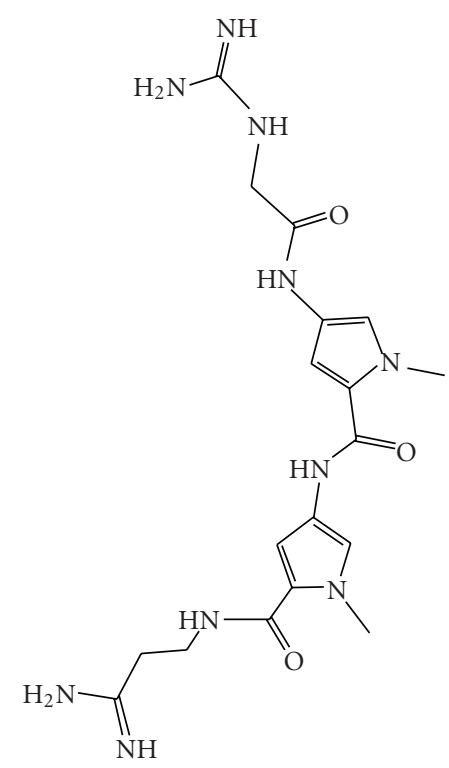

- $2 \mathrm{HCl}$

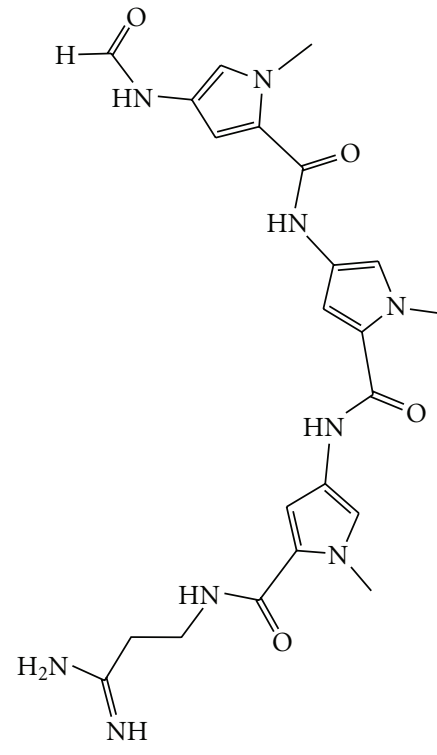

- $\mathrm{HCl}$

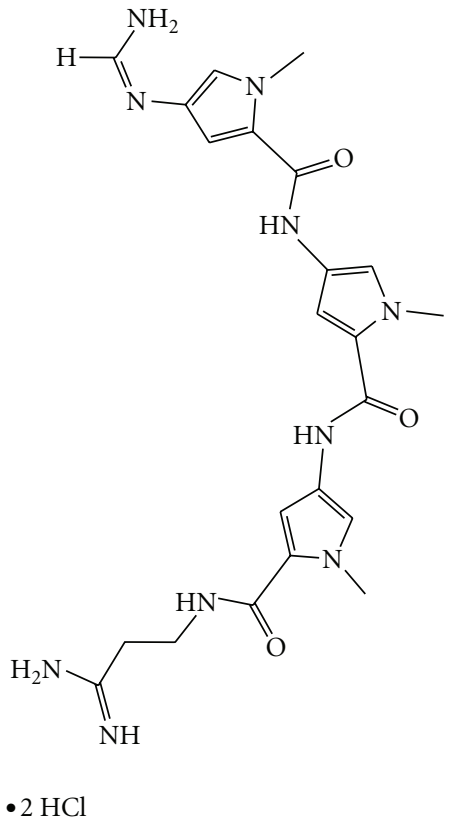

(c)

(a)

(b)

Figure 1: (a) Chemical structures of netropsin, (b) distamycin A, and (c) compound $\mathbf{1}$.

the equilibrium, we applied a spacing of 300 or 400 s between each ligand injection. Heat produced by ligands dilution was evaluated by performing a control experiment, titrating each ligand into the buffer alone. The interaction heat for each injection was calculated after correction for the heat of ligand dilution. The corrected heat values were plotted as a function of the molar ratio, to give the corresponding binding isotherms. The resulting isotherms were fitted to a single set of identical sites model employing a nonlinear least-squares minimisation algorithm to a theoretical titration curve, using the program Bindwork from Calorimetry Science Inc. $\Delta H^{\circ}$ (reaction enthalpy change in $\mathrm{kJ} \mathrm{mol}^{-1}$ ), $K_{b}$ (binding constant in $\mathrm{M}^{-1}$ ), and $n$ (number of binding sites) were the fitting parameters. The Gibbs energy and the entropic contribution were calculated using the relationships $\Delta_{b} G^{\circ}=-R T \ln K_{b}$, $\left(R=8.314 \mathrm{~J} \mathrm{~mol}^{-1} \mathrm{~K}^{-1}, T=298 \mathrm{~K}\right)$ and $-T \Delta_{b} S^{\circ}=\Delta_{b} G^{\circ}-$ $\Delta_{b} H^{\circ}$.

\section{Results}

3.1. NMR Experiments. The addition of compound 1 to the quadruplex $\left[\mathrm{d}(\mathrm{TGGGGT)}]_{4}\right.$ (Figure 2 ) caused gradual changes in the chemical shift and a broadening of DNA proton resonances in the ${ }^{1} \mathrm{H}-\mathrm{NMR}$ spectra. The titration was virtually completed at ligand:DNA ratio of $2: 1$. The four strands resulted to be magnetically equivalent throughout the titration, since no splitting of resonances was observed at any stage. Furthermore, a single set of signals was present for derivative 1 protons throughout the whole NMR titration, which only grew in intensity and did not show any significant change in chemical shift values by increasing ligand concentration.
We also studied the interaction of compound 1 with the DNA duplex d(CGCGAATTCGCG) $)_{2}$ containing the central AATT core, which is considered the specific binding site for distamycin and netropsin, to evaluate a possible selectivity of this analogue. The ${ }^{1} \mathrm{H}-\mathrm{NMR}$ spectrum $(700 \mathrm{MHz}$, $T=25^{\circ} \mathrm{C}$ ) of $\mathrm{d}(\mathrm{CGCGAATTCGCG})_{2}$ turned out to be consistent with the assignment already published by Hare et al. (taking in due account the different temperature at which it has been assigned) [25]. The NMR titration of $\mathrm{d}$ (CGCGAATTCGCG) $)_{2}$ with $\mathbf{1}$ is reported in Figure 3. In this case, most of the DNA resonances turned out to be not affected by the addition of ligand, but some resonances of residues $\mathrm{C}$ 1, A5, A6, T7, T8, C9.

3.2. ITC Experiments. Examples of the raw ITC and integrated heat data for the titration of $[\mathrm{d}(\mathrm{TGGGGT})]_{4}$ quadruplex with netropsin and compound 1 are shown in Figure 4. The ITC data for drugs binding to $[\mathrm{d}(\mathrm{TGGGGT})]_{4}$ indicate, in both cases, the formation of a $2: 1$ (drug:quadruplex) complex with good affinity. The raw data for the titration of drugs with $[\mathrm{d}(\mathrm{TGGGGT})]_{4}$ (insets in Figure 4) indicate an exothermic interaction, based on the positive values observed for the peaks. With each injection of ligand, less and less heat release was observed until constant values were obtained, reflecting, in both cases, a saturable process. The thermodynamic results obtained from fitting the ITC data for netropsin and compound $\mathbf{1}$ binding to [d(TGGGGT) $]_{4}$ are given in Table 1 . The values of the binding constants and the Gibbs energy changes $\left(-34 \mathrm{~kJ} \mathrm{~mol}^{-1}\right.$ and $-36 \mathrm{~kJ} \mathrm{~mol}^{-1}$ for netropsin and compound 1, resp.) indicate that, from a thermodynamic point of view, the interactions with the quadruplex molecule are favoured at $25^{\circ} \mathrm{C}$. 


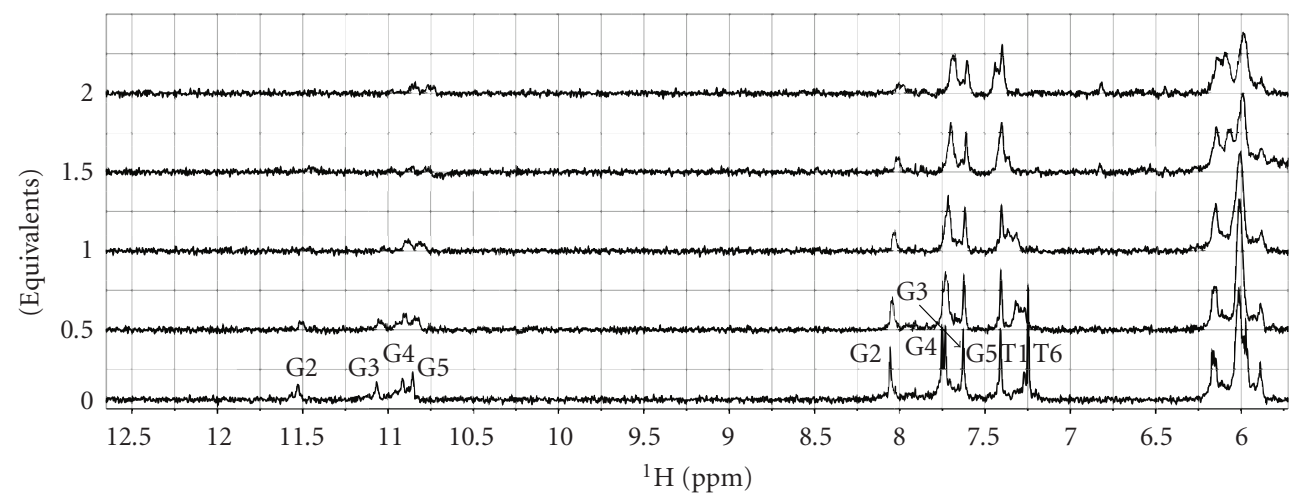

Figure 2: NMR titration of $[\mathrm{d}(\mathrm{TGGGGT})]_{4}$ with derivative $1\left(700 \mathrm{MHz}, T=25^{\circ} \mathrm{C}\right) .2 \mathrm{mM}(8 \mathrm{mM}$ single strand concentration), in $0.2 \mathrm{ml}$ $\left(\mathrm{H}_{2} \mathrm{O} / \mathrm{D}_{2} \mathrm{O} 9: 1\right)$ buffer solution having $10 \mathrm{mM} \mathrm{KH}_{2} \mathrm{PO}_{4}, 70 \mathrm{mM} \mathrm{KCl}, 0.2 \mathrm{mM}$ EDTA, and $\mathrm{pH}$ 7.0. Equivalents of the drug are reported on the left of each spectrum.

TABLE 1: Thermodynamic parameters for the interaction of compound 1 and netropsin with $[\mathrm{d}(\mathrm{TGGGGT})]_{4}$ determined by ITC at $25^{\circ} \mathrm{C}$ and $\mathrm{pH} 7.0$.

\begin{tabular}{lccccc}
\hline Ligand & $n$ & $K_{b}\left(\times 10^{6} \mathrm{M}^{-1}\right)$ & $\Delta_{b} H^{\circ}\left(\mathrm{kJ} \mathrm{mol}^{-1}\right)$ & $T \Delta_{b} S^{\circ}\left(\mathrm{kJ} \mathrm{mol}^{-1}\right)$ & $\left.\Delta_{b} G^{\circ}(\mathrm{kJ} \mathrm{mol})^{-1}\right)$ \\
\hline Compound 1 & $2.0 \pm 0.1$ & $1.9 \pm 0.2$ & $-11.0 \pm 2.0$ & $25 \pm 2$ & $-36 \pm 2$ \\
Netropsin & $2.0 \pm 0.1$ & $1.2 \pm 0.1$ & $-10.6 \pm 1.0$ & $23 \pm 2$ & $-34 \pm 2$ \\
\hline
\end{tabular}

The values of $\Delta H^{\circ}$ and $T \Delta S^{\circ}$ show that in both cases the interactions are associated with a favourable binding enthalpy $\left(-10.6 \mathrm{~kJ} \mathrm{~mol}^{-1}\right.$ and $-11.0 \mathrm{~kJ} \mathrm{~mol}^{-1}$ for netropsin and compound 1 , resp.), however, the binding processes are always entropically driven $\left(23 \mathrm{~kJ} \mathrm{~mol}^{-1}\right.$ and $25 \mathrm{~kJ} \mathrm{~mol}^{-1}$ for netropsin and compound $\mathbf{1}$, resp.).

Finally, we also performed experiments on the interaction of compound $\mathbf{1}$ with the DNA duplex d(CGCGAATTCGCG) $)_{2}$ containing the central AATT core, which is considered the specific binding site for distamycin and netropsin, to evaluate if the structural modifications of compound 1 influence the binding. An example of the raw ITC data for the titration of the d(CGCGAATTCGCG) $)_{2}$ duplex with compound $\mathbf{1}$ is shown in Figure 5. Resolvable binding isotherm was never obtained for the interaction of compound 1 with duplex using any combination of reactant concentrations, suggesting low affinity of the molecule for the investigated duplex.

\section{Discussion}

Distamycin and netropsin have been recognized for decades as nonintercalative DNA binding ligands that show specificity for the minor groove of dA - dT base pairs $[22,26]$. The binding of the drugs to duplex DNA involves an electrostatic component from the cationic ends, hydrogen bonds from the amide $\mathrm{NH}$ groups, and van der Waals interactions with the wall of the groove. Some years ago, NMR studies indicated that, depending on DNA sequence, some binding sites can accommodate two distamycin molecules side-by-side in an antiparallel orientation [27]. In this $2: 1$ complex each ligand molecule preserves all the molecular recognition elements of groove binders. In contrast to distamycin, the dication netropsin binds only as a single molecule per binding site, suggesting that the side-by-side arrangement of two molecules is inhibited by charge repulsions. Both drugs have been shown little or no affinity for single-stranded DNA or RNA or for double-stranded RNA or DNA-RNA hybrids as well as they do not bind to the A helix or the left-handed ZDNA $[26,28]$.

Surprisingly, distamycin A has recently been shown to interact also with four-stranded parallel DNA quadruplexes $[12,29,30]$. Particularly, we have proved, by using NMR and ITC methodology, that distamycin is able to interact with the quadruplex [d(TGGGGT) $]_{4}$. We showed that four ligand molecules bind as antiparallel dimers to the quadruplex in two opposite grooves, establishing hydrogen bonds with the guanine bases and strong coulombic interactions between the positively charged amidinium moiety of the ligand and the backbone phosphate groups of the quadruplex [12]. On the other hand, netropsin turned out to possess a lower affinity (NMR data) towards the quadruplex [d(TGGGGT) $]_{4}$ [29], even if, till now, this has never been confirmed by ITC. Interestingly, it seems that netropsin is not able to bind the quadruplex in dimeric form, most probably due to the doubly charged nature of molecule that prevents a side-byside arrangement into the grooves.

In order to evaluate the binding properties of derivative 1, and to perform a direct comparison with the binding behaviour of distamycin A and netropsin, [d(TGGGGT) $]_{4}$ has been titrated with 1 at the same experimental conditions (buffer, temperature, DNA concentration) used for distamycin A [29]. As far as distamycin A is concerned, below 2:1 ligand: quadruplex stoichiometry, the addition of distamycin A to [d(TGGGGT) $]_{4}$ caused gradual changing in chemical shift of the signal of the quadruplex, whereas 


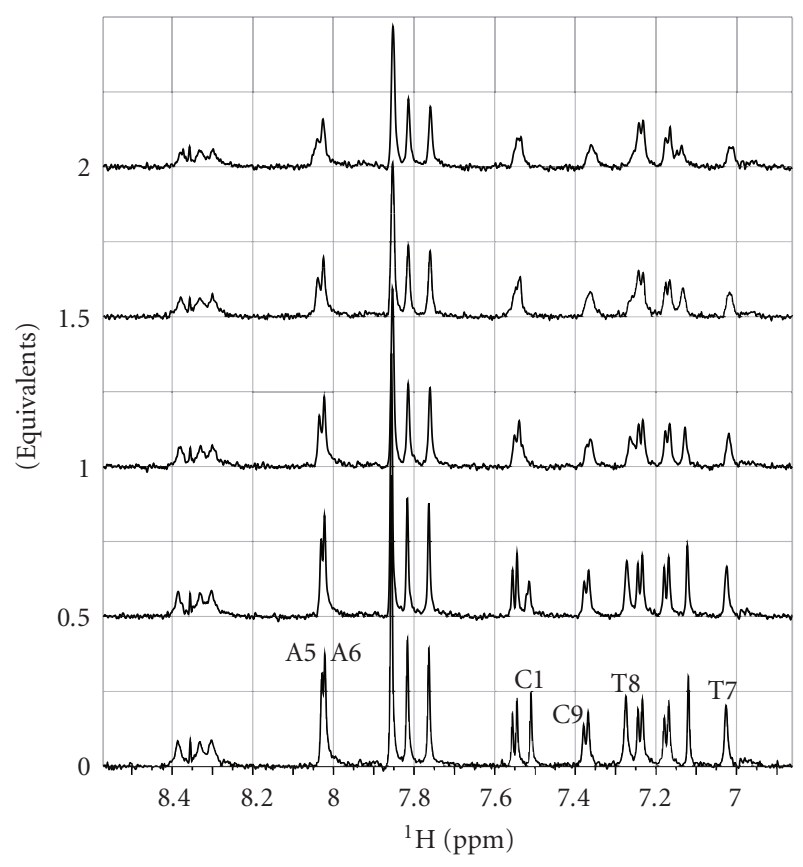

FIgURE 3: Expanded region of the ${ }^{1} \mathrm{H}-\mathrm{NMR}$ titration of $\mathrm{d}(\text { CGCGAATTCGCG })_{2}$ with derivative $1\left(700 \mathrm{MHz}, T=25^{\circ} \mathrm{C}\right)$. $1 \mathrm{mM}$ (4 mM single strand concentration), in $0.2 \mathrm{ml}\left(\mathrm{H}_{2} \mathrm{O} / \mathrm{D}_{2} \mathrm{O}\right.$ 9:1) buffer solution having $10 \mathrm{mM} \mathrm{KH}_{2} \mathrm{PO}_{4}, 70 \mathrm{mM} \mathrm{KCl}, 0.2 \mathrm{mM}$ EDTA, and pH 7.0. Equivalents of the drug are reported on the left of each spectrum. Residues that are slightly affected by the binding are indicated.

further addition of drug caused the appearance of a new set of proton signals, whose intensities rose by increasing the amount of drug with the concomitant falling off of the original signals which completely disappeared at a ratio of $4: 1$ drug-DNA. Differently, the NMR titration profile of 1 (Figure 2) turned out to be very different from that observed in the case of distamycin A and clearly suggests that derivative 1 binds the quadruplex in a fast process on the NMR time scale, very similarly to the NMR titration profile observed for netropsin [29].

In order to preliminarily evaluate the binding site of derivative 1, a comparison of resonances of some protons of the uncomplexed DNA and the complexed one has been done. In particular, we report the $\Delta \delta$ values (chemical shifts of the complex minus free DNA) of aromatic, methyl, and imino protons in Figure 6. Interestingly, all analyzed DNA resonances shifted. Nevertheless, the signal of the protons of T1 residue shifted the least, whereas the ones of residue T6 the more. In any case, a general shift of the aromatic and imino signals could be observed also for the G2, G3, G4, and G5. This means that, basically, derivative $\mathbf{1}$ is able to recognize most of the molecule surface.

On the other hand, the addition of 1 to d(CGCGAATTCGCG) $)_{2}$ affected only slightly some DNA resonances. In particular, at 2:1 (drug/DNA) molar ratio the aromatic protons of residue $\mathrm{C} 1, \mathrm{~A} 5, \mathrm{~A} 6, \mathrm{~T} 7, \mathrm{~T} 8, \mathrm{C} 9$ residues underwent to a shift of only $0.03,0.01,0.01,0.02,0.03$, and $0.01 \mathrm{ppm}$, respectively, indicating a very poor affinity towards the dodecamer.

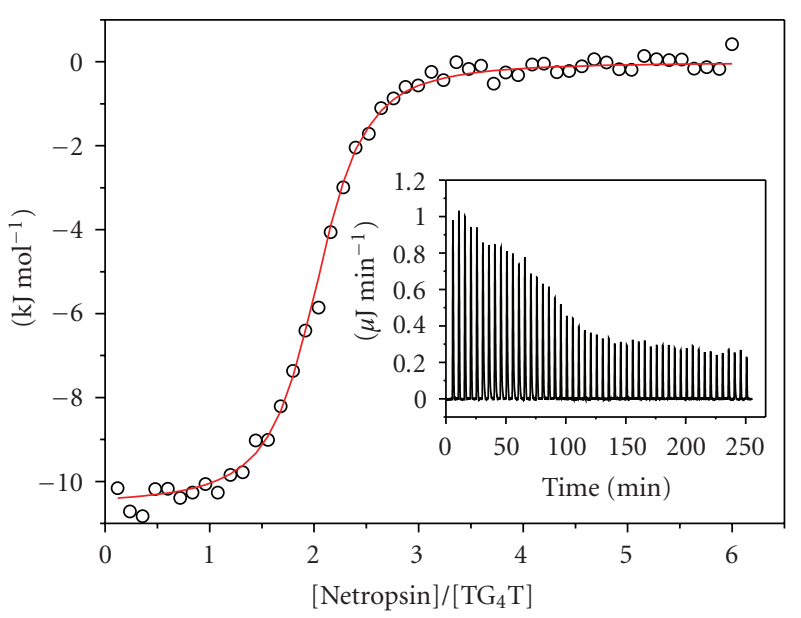

(a)

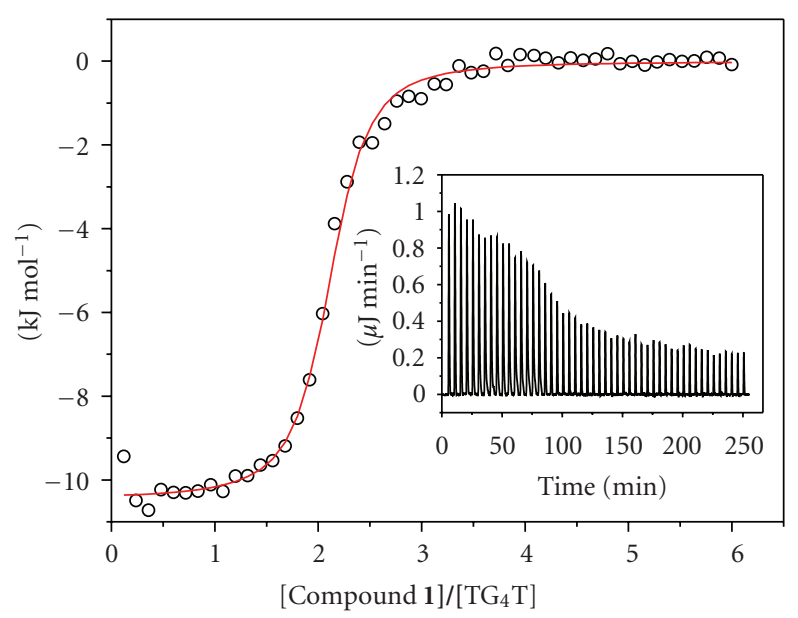

(b)

FIGURE 4: Raw ITC data (insets) and binding isotherms for titration of $[\mathrm{d}(\mathrm{TGGGGT})]_{4}$ with netropsin (a) and with compound $\mathbf{1}$ (b).

ITC is an useful methodology for a complete understanding of drug-DNA interactions, and it has been applied many times to determine the thermodynamic properties of drug-quadruplex interactions [31]. Indeed, ITC is the only technique that directly measures the binding enthalpy change for the formation of a complex, allowing the free energy change to be dissected into the enthalpic and entropic contribution to the association process. This reveals the nature of the forces that drive the binding and can provide insight into the nature of the intermolecular contacts formed and even into changes in solvation [19]. The understanding of those factors can be helpful in both screening among various drugs and optimizing the drug-target interactions, to direct the design of new drugs.

ITC experiments reveal that both compound 1 and netropsin bind to the investigated quadruplex. The thermodynamic profiles of the two drug-quadruplex interactions are qualitatively similar and in both cases the stoichiometry observed is 2:1 (drug:quadruplex). Interestingly, the thermodynamic parameters determined by ITC (Table 1) 


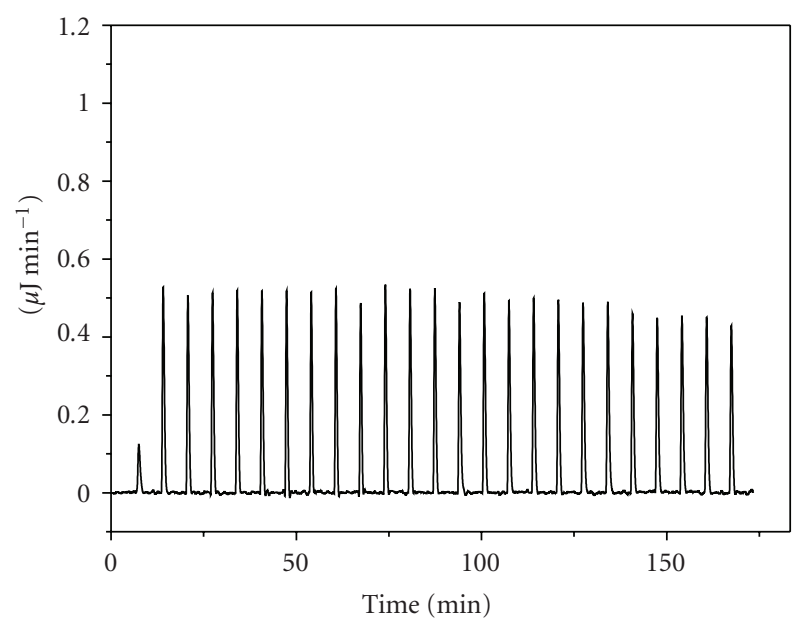

FIGURE 5: Raw ITC data for titration of d(CGCGAATTCGCG) duplex with compound 1 . With each injection of ligand, constant heat release was observed due only to ligand dilution.

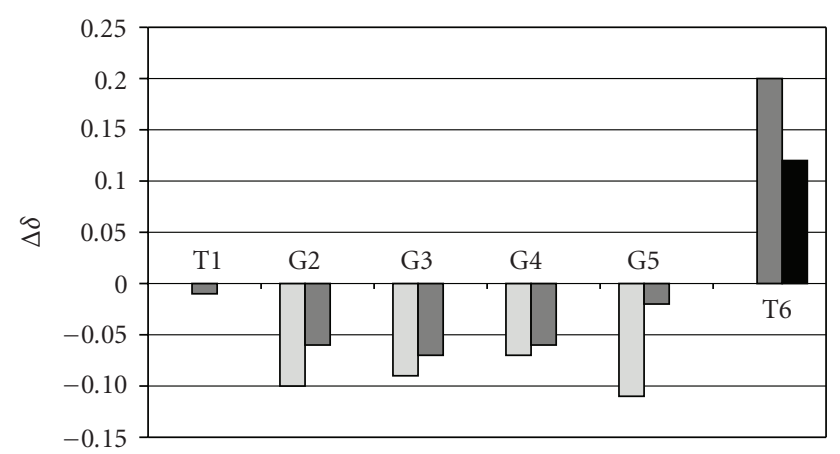

FIGURE 6: Difference in chemical shifts $(\Delta \delta)$ of DNA upon binding of 1 (complex 1: DNA minus DNA alone) with [d(TGGGGT) $]_{4} . \Delta \delta$ values are reported for imino (light gray), aromatic (dark gray), and methyl (black) protons.

indicate that the association reactions of both ligands with $[\mathrm{d}(\mathrm{TGGGGT})]_{4}$ are entropically driven processes, even if the direct ITC measurements of the binding enthalpy change indicate that also the enthalpic contribution favours the associations. In the case of the interaction of distamycin with $[\mathrm{d}(\mathrm{TGGGGT})]_{4}$, similar results were obtained (entropically driven process with a small favourable enthalpic contribution), except for stoichiometry and magnitude of binding constant.

As reported in the literature many times, a groove binding is generally characterized by a large favourable increase of entropy and a small favourable or unfavourable enthalpy change [32]. This could be mainly due to the fact that the association reaction is driven in large part by a hydrophobic effect and the favorable entropy is derived from the release of bound water molecules from the DNA and drug upon complex formation as well as release of counterions upon binding of cationic ligands. Interestingly, the entropically driven interactions of the compound $\mathbf{1}$ and of netropsin suggest a groove-binding mechanism also in these cases, as observed for distamycin A. Additionally, in contrast to many other groove binders, netropsin binding to duplex DNA is accom- panied on average by a favourable enthalpy change. This is the result of both electrostatic interactions and hydrogen bonds formation between netropsin molecule and DNA. A favourable enthalpy change is observed by ITC for netropsin binding to $[\mathrm{d}(\mathrm{TGGGGT})]_{4}$ as well as for compound 1, suggesting once again a similar groove binding mode.

As stated before, the stoichiometry obtained for the association of the investigated ligands to [d(TGGGGT) $]_{4}$ is $2: 1$ (drug: quadruplex), while for distamycin a stoichiometry of $4: 1$ was found. This finding is consistent with the observation that the distamycin molecules are able to bind as dimers, while netropsin (and probably also compound $\mathbf{1}$ ) is not able to dimerise because of charge repulsions. Notably, in the distamycin-[d(TGGGGT) $]_{4}$ complex, each distamycin dimer expands its bounded groove (similarly to that observed with duplex DNA), with concomitant reduction of the size of the adjacent ones, preventing a further interaction with other ligand molecules. In the binding of netropsin to duplex DNA the minor groove is widened by $0.5-2.0 \AA$ by the entry of the drug [16]. We can speculate that the binding of the two netropsin molecules to $[\mathrm{d}(\mathrm{TGGGGT})]_{4}$ could similarly expand the bounded grooves, reducing the size of the adjacent ones and preventing more interactions.

The experiments on the interaction of compound 1 with the DNA duplex d(CGCGAATTCGCG) $)_{2}$ show that compound $\mathbf{1}$ has a poor affinity for the investigated duplex, while it is well known that both distamycin and netropsin have a high binding constant (between $10^{6}$ and $10^{8} \mathrm{M}^{-1}$ ) for that target containing the specific binding site. This finding demonstrates that the structural modifications of compound 1 decrease the affinity of the ligand toward the duplex, enhancing the selectivity.

\section{Conclusions}

The combination of calorimetric and NMR methodologies has enabled us to demonstrate that netropsin and compound $\mathbf{1}$ are able to bind to $[\mathrm{d}(\mathrm{TGGGGT})]_{4}$ with good affinity, forming, in both cases, a 2:1 (drug: quadruplex) complex.

Very interestingly, this study shows that the structural modifications of compound $\mathbf{1}$ do not influence, compared to netropsin, the interaction with the quadruplex, but such modifications decrease the affinity of the ligand toward the duplex, enhancing the selectivity.

\section{Acknowledgments}

This work is supported by the Italian Institute of Technology (IIT), Italian M.U.R.S.T. (P.R.I.N. 2007), Regione Campania (L.41, L.5), and by the COST Action MP0802. The authors are grateful to "Centro di Servizio Interdipartimentale di Analisi Strumentale", C.S.I.A.S., for supplying NMR facilities.

\section{References}

[1] S. Burge, G. N. Parkinson, P. Hazel, A. K. Todd, and S. Neidle, "Quadruplex DNA: sequence, topology and structure," Nucleic Acids Research, vol. 34, no. 19, pp. 5402-5415, 2006. 
[2] J. L. Huppert and S. Balasubramanian, "Prevalence of quadruplexes in the human genome," Nucleic Acids Research, vol. 33, no. 9, pp. 2908-2916, 2005.

[3] J. E. Johnson, J. S. Smith, M. L. Kozak, and F. B. Johnson, "In vivo veritas: using yeast to probe the biological functions of Gquadruplexes," Biochimie, vol. 90, no. 8, pp. 1250-1263, 2008.

[4] Y. Qin and L. H. Hurley, "Structures, folding patterns, and functions of intramolecular DNA G-quadruplexes found in eukaryotic promoter regions," Biochimie, vol. 90, no. 8, pp. 1149-1171, 2008.

[5] S. Neidle and G. N. Parkinson, "The structure of telomeric DNA," Current Opinion in Structural Biology, vol. 13, no. 3, pp. 275-283, 2003.

[6] G. Laughlan, A. I. H. Murchie, D. G. Norman, et al., "The high-resolution crystal structure of a parallel-stranded guanine tetraplex," Science, vol. 265, no. 5171, pp. 520-524, 1994.

[7] N. W. Kim, M. A. Piatyszek, K. R. Prowse, et al., "Specific association of human telomerase activity with immortal cells and cancer," Science, vol. 266, pp. 2011-2015, 1994.

[8] J. W. Shay and W. E. Wright, "Telomerase therapeutics for cancer: challenges and new directions," Nature Reviews Drug Discovery, vol. 5, no. 7, pp. 577-584, 2006.

[9] B. Pagano and C. Giancola, "Energetics of quadruplex-drug recognition in anticancer therapy," Current Cancer Drug Targets, vol. 7, no. 6, pp. 520-540, 2007.

[10] J.-F. Riou, "G-quadruplex interacting agents targetting the telomeric G-overhang are more than simple telomerase inhibitors," Current Medicinal Chemistry, vol. 4, no. 5, pp. 439-443, 2004.

[11] S. Neidle, "The structures of quadruplex nucleic acids and their drug complexes," Current Opinion in Structural Biology, vol. 19, no. 3, pp. 239-250, 2009.

[12] L. Martino, A. Virno, B. Pagano, et al., "Structural and thermodynamic studies of the interaction of distamycin A with the parallel quadruplex structure $\left[\mathrm{d}(\mathrm{TGGGGT)}]_{4}\right.$," Journal of the American Chemical Society, vol. 129, no. 51, pp. 16048-16056, 2007.

[13] N. Zaffaroni, S. Lualdi, R. Villa, et al., "Inhibition of telomerase activity by a distamycin derivative: effects on cell proliferation and induction of apoptosis in human cancer cells," European Journal of Cancer, vol. 38, no. 13, pp. 17921801, 2002.

[14] B. Pagano, A. Virno, C. A. Mattia, L. Mayol, A. Randazzo, and C. Giancola, "Targeting DNA quadruplexes with distamycin A and its derivatives: an ITC and NMR study," Biochimie, vol. 90, no. 8, pp. 1224-1232, 2008.

[15] B. Pagano, C. A. Mattia, A. Virno, A. Randazzo, L. Mayol, and C. Giancola, "Thermodynamic analysis of quadruplex DNAdrug interaction," Nucleosides, Nucleotides and Nucleic Acids, vol. 26, no. 6-7, pp. 761-765, 2007.

[16] M. L. Kopka, C. Yoon, D. Goodsell, P. Pjura, and R. E. Dickerson, "The molecular origin of DNA-drug specificity in netropsin and distamycin," Proceedings of the National Academy of Sciences of the United States of America, vol. 82, no. 5, pp. 1376-1380, 1985.

[17] M. L. Kopka, C. Yoon, D. Goodsell, P. Pjura, and R. E. Dickerson, "Binding of an antitumor drug to DNA netropsin and C-G-C-G-A-A-T-T- ${ }^{\mathrm{Br}} \mathrm{C}-\mathrm{G}-\mathrm{C}-\mathrm{G}$," Journal of Molecular Biology, vol. 183, no. 4, pp. 553-563, 1985.

[18] L. A. Marky and K. J. Breslauer, "Origins of netropsin binding affinity and specificity: correlations of thermodynamic and structural data," Proceedings of the National Academy of Sciences of the United States of America, vol. 84, no. 13, pp. 4359-4363, 1987.

[19] I. Haq, "Thermodynamics of drug-DNA interactions," Archives of Biochemistry and Biophysics, vol. 403, no. 1, pp. 1$15,2002$.

[20] C. R. Cantor, M. M. Warshaw, and H. Shapiro, "Oligonucleotide interactions. 3. Circular dichroism studies of the conformation of deoxyoligonucleotides," Biopolymers, vol. 9, no. 9, pp. 1059-1077, 1970.

[21] G. Di Pietro, G. Giannini, E. M. Iafrate, et al., " $N$-formimidoyl analogues of distamycin," Journal of the Chemical Society, Perkin Transactions 1, no. 12, pp. 1333-1335, 1996.

[22] D. Rentzeperis, L. A. Marky, T. J. Dwyer, B. H. Geierstanger, J. G. Pelton, and D. E. Wemmer, "Interaction of minor groove ligands to an AAATT/AATTT site: correlation of thermodynamic characterization and solution structure," Biochemistry, vol. 34, no. 9, pp. 2937-2945, 1995.

[23] T. L. Hwang and A. J. Shaka, "Water suppression that works. Excitation sculpting using arbitrary wave-forms and pulsedfield gradients," Journal of Magnetic Resonance A, vol. 112, pp. 275-279, 1995.

[24] C. Dalvit, "Efficient multiple-solvent suppression for the study of the interactions of organic solvents with biomolecules," Journal of Biomolecular NMR, vol. 11, no. 4, pp. 437-444, 1998.

[25] D. R. Hare, D. E. Wemmer, S.-H. Chou, G. Drobny, and B. R. Reid, "Assignment of the non-exchangeable proton resonances of d(C-G-C-G-A-A-T-T-C-G-C-G) using two-dimensional nuclear magnetic resonance methods," Journal of Molecular Biology, vol. 171, no. 3, pp. 319-336, 1983.

[26] R. M. Wartell, J. E. Larson, and R. D. Wells, "Netropsin. A specific probe for AT regions of duplex deoxyribonucleic acid," The Journal of Biological Chemistry, vol. 249, no. 21, pp. 67196731, 1974.

[27] J. G. Pelton and D. E. Wemmer, "Binding modes of distamycin A with d(CGCAAATTTGCG) $)_{2}$ determined by twodimensional NMR," Journal of the American Chemical Society, vol. 112, no. 4, pp. 1393-1399, 1990.

[28] C. Zimmer, "Effects of the antibiotics netropsin and distamycin A on the structure and function of nucleic acids," Progress in Nucleic Acid Research and Molecular Biology, vol. 15, pp. 285-318, 1975.

[29] A. Randazzo, A. Galeone, and L. Mayol, "1 H-NMR study of the interaction of distamycin A and netropsin with the parallel stranded tetraplex $[\mathrm{d}(\mathrm{TGGGGT})]_{4}$," Chemical Communications, vol. 11, pp. 1030-1031, 2001.

[30] M. J. Cocco, L. A. Hanakahi, M. D. Huber, and N. Maizels, "Specific interactions of distamycin with G-quadruplex DNA," Nucleic Acids Research, vol. 31, no. 11, pp. 2944-2951, 2003.

[31] B. Pagano, C. A. Mattia, and C. Giancola, "Applications of isothermal titration calorimetry in biophysical studies of Gquadruplexes," International Journal of Molecular Sciences, vol. 10, no. 7, pp. 2935-2957, 2009.

[32] J. B. Chaires, "A thermodynamic signature for drug-DNA binding mode," Archives of Biochemistry and Biophysics, vol. 453, no. 1, pp. 24-29, 2006. 

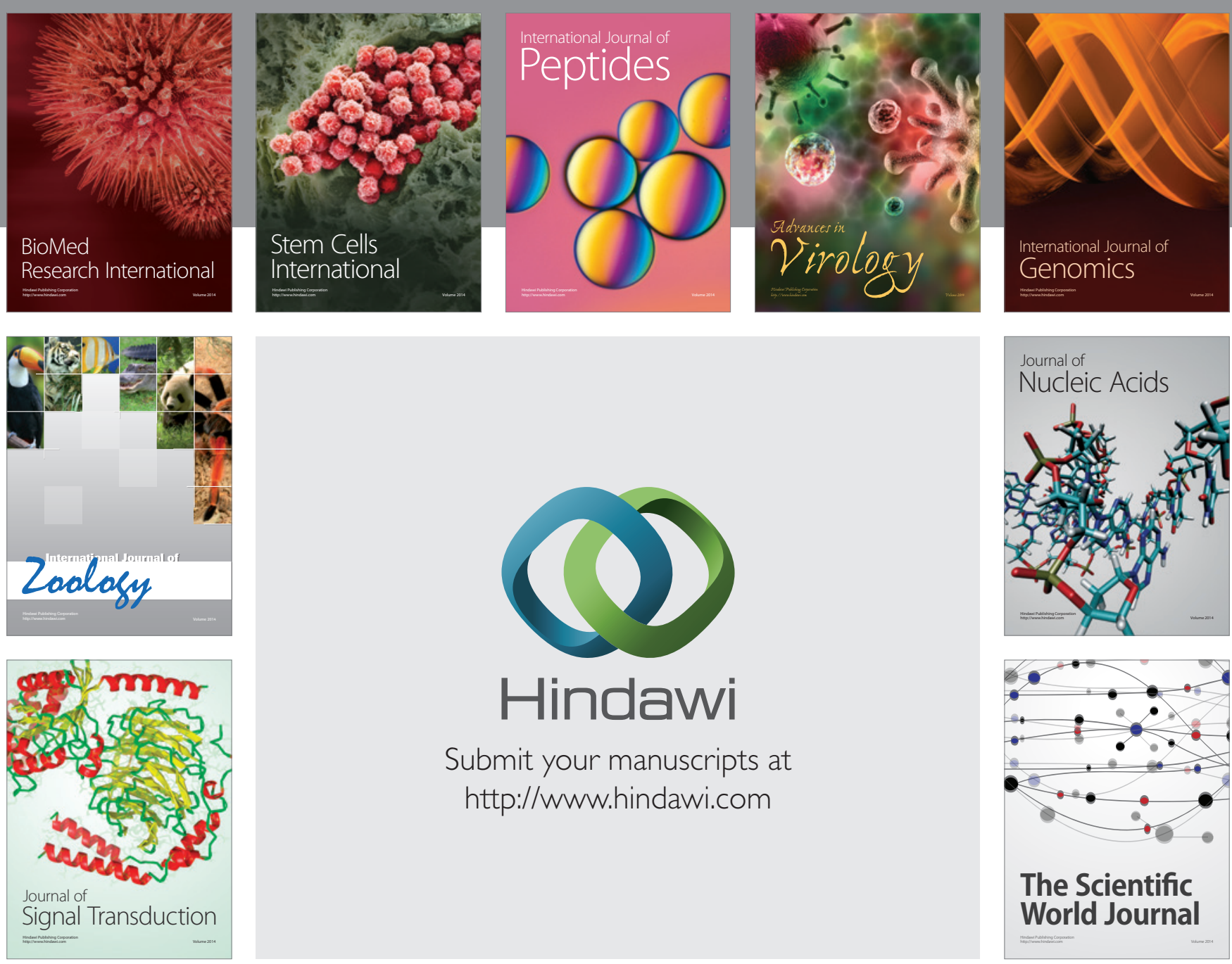

Submit your manuscripts at

http://www.hindawi.com
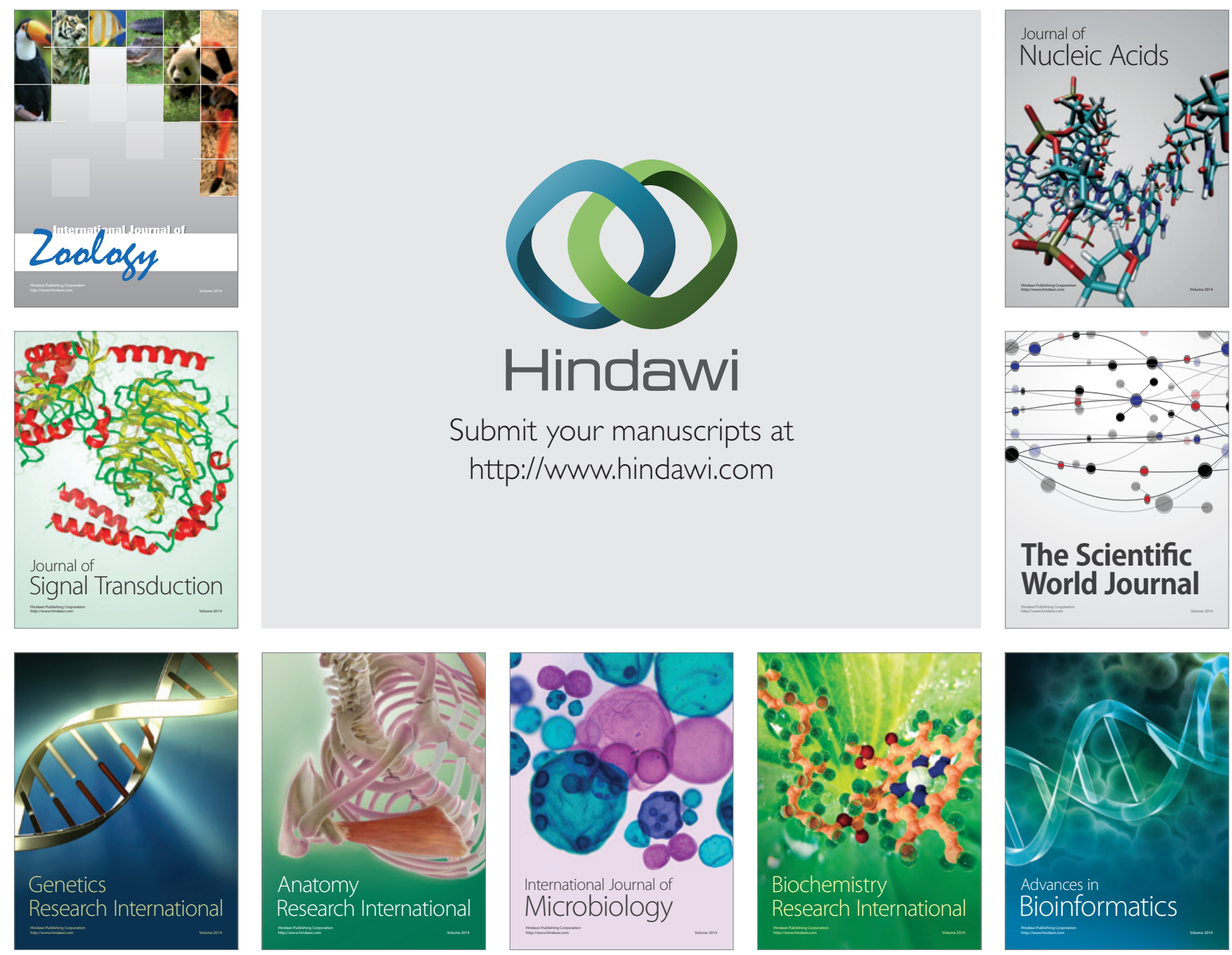

The Scientific World Journal
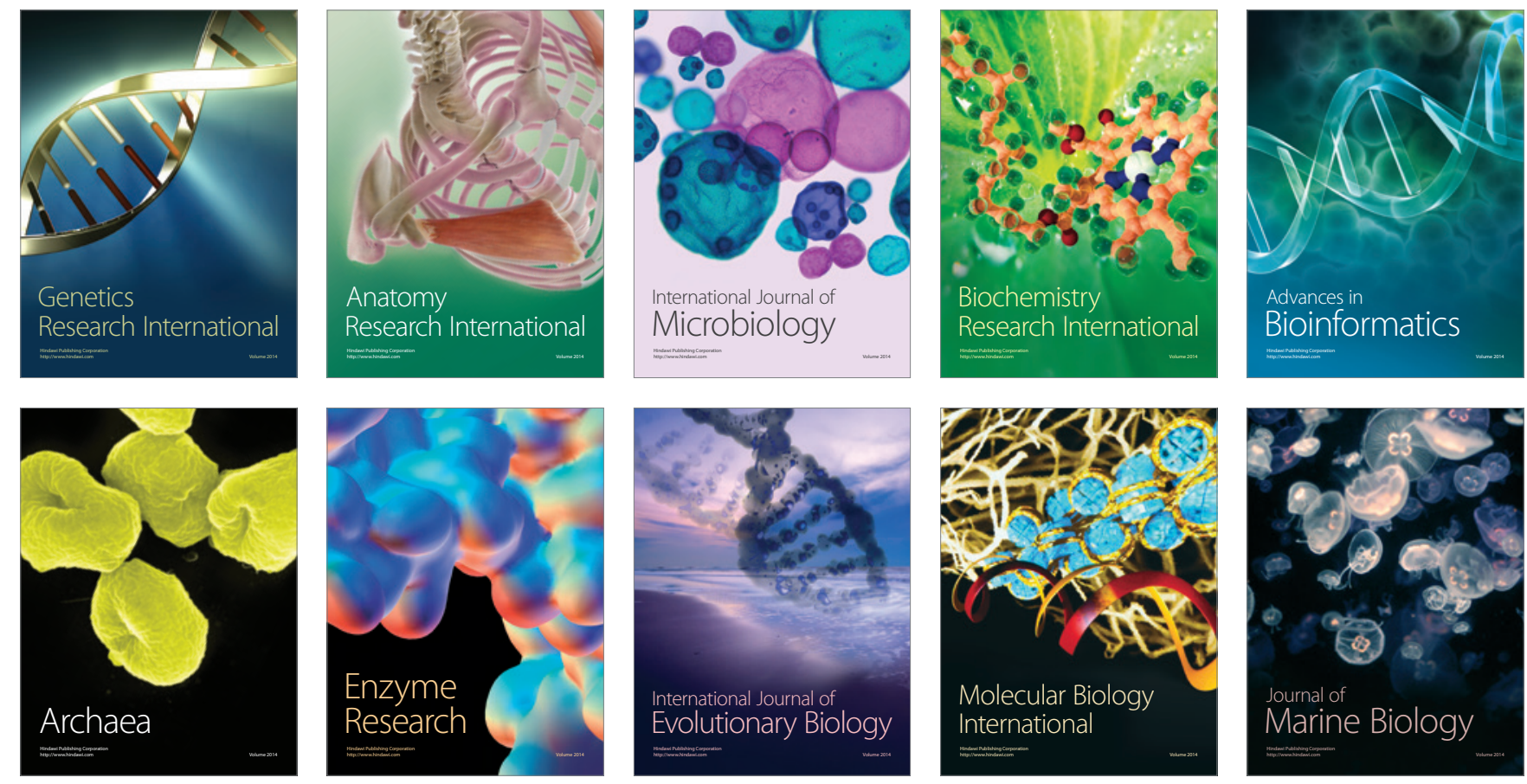